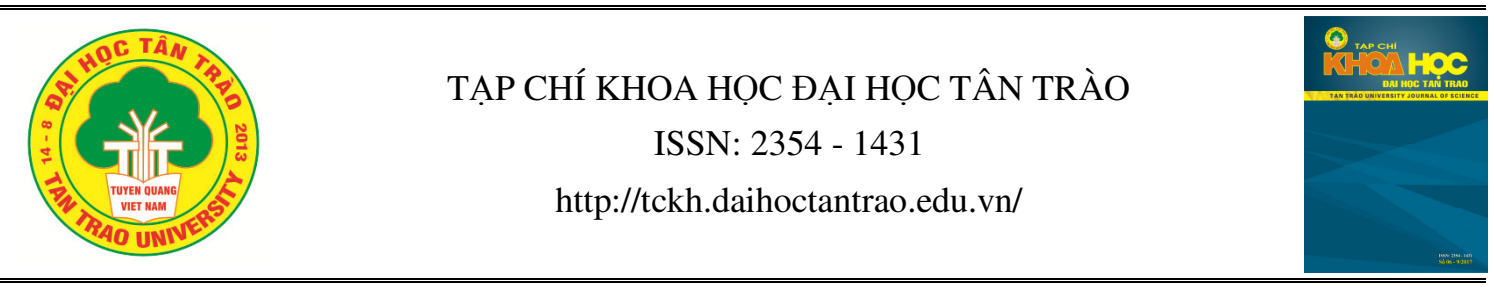

\title{
Khử phân kỳ trong biên độ tán xạ toàn phần của quá trình rã $h \rightarrow \mu T$ trong mô hình Zee-Babu
}

\author{
Trần Trung Hiếu $u^{a}$, Nguyễn Thị Thu Vân ${ }^{a}$, Dưong Thị Kiều Tüb, Hà Thanh Hùng ${ }^{a}{ }^{*}$, Trịnh Phi Hiệp $p^{c}$ \\ ${ }^{a}$ Trường Đại học Sư phạm Hà Nội 2 \\ ${ }^{b}$ Truoòng Sỹ quan Pháo binh \\ ${ }^{c}$ TruòngĐại học Tân Trào \\ *Email: hathanhhung@hpu2.edu.vn
}

\section{Thông tin bài viết}

Ngày nhận bài:

26/03/2018

Ngày duyệt đăng:

$12 / 6 / 2018$

Tù khoá:

Các hàm $P V$, khủ phân kỳ, mô hìh Zee-Babu, vi phạm số lepton thế hệ, rã Higgs boson.

\section{Tóm tắt}

Mô hình Zee-Babu là sự tiếp nối của mô hình Zee được mở rộng từ mô hình chuẩn để giải quyết khối lượng và sự trộn lẫn neutrino. Khác với mô hình Zee, phần vô hướng của mô hình Zee-Babu được thêm vào đơn giản hơn, do đó vấn đề neutrino được giải quyết một cách tự nhiên. Các kênh rã của Higgs boson đã và đang được thực nghiệm kiểm chứng. Các tương tác mới trong mô hình Zee-Babu góp phần làm tăng tín hiệu của các kênh rã Higgs vi phạm số lepton. Nghiên cứu các kênh rã này giúp giới hạn các vùng không gian tham số của mô hình. Đó cũng là cơ sở để đưa ra các hiệu ứng vật lý mới.

\section{Giới thiệu}

Các kênh rã vi phạm số lepton đang được nghiên cứu sâu rộng trong các mô hình mở rộng mô hình chuẩn dựa vào số liệu thực nghiệm liên tục được cập nhật. Năm 2016, giới hạn thực nghiệm chính xác nhất của các kênh rã vi phạm số lepton của các lepton mang điện đã được đưa ra tại phòng thí nghiệm $B A B A R$ và $B E L L E$ [2], giới hạn của các kênh rã Higgs vi phạm số lepton cũng được đưa ra tại phòng thí nghiệm CMS\&ATLAS [1]. Việc sử dụng các hàm PV (Pasarino-Veltman) để biểu diễn các biên độ tán xạ có thể thay thế cho phần mềm Looptools và áp dụng được cho nhiều mô hình [5,6]. Mô hình ZeeBabu khi thêm vào các hạt mới đã làm xuất hiện các tương tác vi phạm số lepton [3,8,9]. Nghiên cứu các kênh rã vi phạm số lepton của lepton mang điện trong mô hình này giúp ta giải thích được sự trộn lẫn của các Meson $\left(K^{0}, B^{0}\right)$ [8] cũng như giới hạn được các vùng không gian tham số và đưa ra khối lượng của các Higgs mang điện [3,7]. Đặc biệt, với các kênh rã vi phạm số lepton của các Higgs giúp chúng ta giải thích được khối lượng và sự trộn lẫn của neutrino, đưa ra giới hạn của góc trộn $\left(\theta_{13}\right)$ [4].
Bài báo này nghiên cứu kênh rã $h \rightarrow \mu \tau$ trong mô hình Zee-Babu. Từ các đỉnh tương tác vi phạm số lepton do đóng góp của các hạt mới, tất cả các giản đồ Feynman của kênh rã này được đưa ra. Việc biểu diễn các biên độ tán xạ theo các hàm PV (PasarinoVeltman) giúp chỉ ra phần phân kỳ trong biên độ toàn phần của quá trình rã $h \rightarrow \mu \tau$ hoàn toàn bị triệt tiêu.

\section{Nội dung}

\subsection{Mô hình Zee-Babu}

Mô hình Zee-Babu có nhóm đối xứng chuẩn giống như mô hình chuẩn (nhóm 3-2-1). Để giải quyết vấn đề neutrino mô hình này được thêm vào các hạt mới. Các hạt mới thêm vào so với mô hình chuẩn là các hạt vô hướng thuộc đơn tuyến của nhóm $S U(2)_{L}$. Do đó, phần sắp xếp các hạt trong mô hình được biểu thị như sau:

Lepton: Các hạt phân cực trái và phân cực phải lần lượt được xếp vào lưỡng tuyến và đơn tuyến của nhóm $S U(2)_{L}$

$$
\Psi_{a L}=\left(\begin{array}{l}
v_{a} \\
e_{a}
\end{array}\right)_{L} \sim(1,2,-1), e_{\mathrm{Ra}} \sim(1,1,-2)
$$


Quark: Các hạt phân cực trái được xếp vào lưỡng tuyến, còn các hạt phân cực phải xếp vào đơn tuyến của nhóm $S U(2)_{L}$

$$
\begin{gathered}
Q_{a L}=\left(\begin{array}{l}
u_{a} \\
d_{a}
\end{array}\right)_{L} \sim(3,2,1 / 3) ; \quad u_{a R} \sim(3,1,4 / 3) \\
; d_{a R} \sim(3,1,-2 / 3)(2)
\end{gathered}
$$

Với $a=1,2,3$ tương ứng là chỉ số thế hệ, còn bộ ba số trong ngoặc đơn tương ứng là số lượng tử của các nhóm $S U(3)_{C}, S U(2)_{L}, U(1)_{Y}$

Vô hướng: Trong mô hình Zee-Babu có ba đa tuyến vô hướng bao gồm một lưỡng tuyến của nhóm $S U(2)_{L}$ và hai đơn tuyến Higgs một mang điện đơn và một mang điện đôi.

$$
\begin{aligned}
& \varphi=\left(\varphi^{+}, \varphi^{0}\right)^{T} \sim(1,2,1), \\
& H^{+} \sim(1,1,2), K^{++} \sim(1,1,4)
\end{aligned}
$$

Thành phần lưỡng tuyến được biểu diễn theo giá trị trung bình chân không v như sau:

$$
\varphi=\left(\begin{array}{c}
\varphi^{+} \\
\frac{1}{\sqrt{2}}\left(\mathrm{v}+\varphi_{R}^{0}+i \varphi_{I}^{0}\right)
\end{array}\right)
$$

Thế Higgs đặc trưng cho mô hình Zee-Babu là: $V_{Z B}=\mu_{H} H^{2}+\mu_{K} K^{2}+\lambda_{H} H^{4}+\lambda_{K} K^{4}+\lambda_{H K} H^{2} K^{2}$ $\mathrm{m}$ 盂 $+\lambda_{1} H^{2} \varphi^{+} \varphi+\lambda_{2} K^{2} \varphi^{+} \varphi+\left(\mu H^{2} K^{++}+\right.$h.c $)$

Khối lượng các Higgs được xác định từ điều kiện cực tiểu thế Higgs.

$$
m_{h}^{2}=\lambda v^{2} ; m_{H}^{2}=\lambda_{H}{ }^{2}+\lambda_{1} v^{2} ; m_{K}{ }^{2}=\lambda_{K}{ }^{2}+\lambda_{2} v^{2}(6)
$$

Trong đó: $h$ là Higgs trung hòa (đồng nhất với $\varphi_{R}^{0}$ ), $H^{ \pm}$là Higgs mang điện đơn, $K^{ \pm \pm}$là Higgs mang điện đôi.

\subsection{Các giản đồ Feyman của quá trình rã $h \rightarrow$}

\section{$\mu$ Ttrong mô hình Zee-Babu}

Lagrangian toàn phần của mô hình Zee-Babu là: [3,7]

$$
L_{t o t}=L_{S M}+L_{Z B}
$$

Trong đó, $L_{S M}$ là phần Lagrangian giống của mô hình chuẩn:

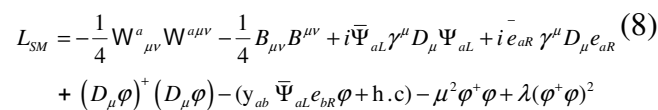

$L_{Z B}$ là phần Lagrangian đặc trưng cho mô hình Zee-Babu:

$L_{Z B}=D_{\mu} H^{+} D^{\mu} H+D_{\mu} K^{+} D^{\mu} K+f_{a b} C\left(\bar{\Psi}_{a L}\right)\left(i \sigma_{2}\right) \Psi_{b L} H^{+}$

एس山llit $g_{a b} C\left(\bar{e}_{a R}\right) e_{b R} K^{++}+h . c+V_{Z B}$

Mối liên hệ giữa các neutrino ban đầu với các trạngthái vật lý của neutrino được liên hệ thông qua ma trận chuyển cơ sở $U_{a b}$. Tức là:

$$
\overline{\boldsymbol{V}}_{a L}^{\prime}=U_{a b}{\overline{\nu_{b L}}}_{b L} ; \nu_{a L}^{\prime}=U_{a b}^{*} \nu_{b L}
$$

\begin{tabular}{|c|c|c|c|c|c|c|c|c|}
\hline Đinh & $\bar{v}_{a} W_{\mu}^{+} e_{b}$ & $h W_{\mu}^{+} W_{v}^{-}$ & $h \bar{w}_{a} \nu_{a}$ & $h \bar{e}_{a} e_{a}$ & $h H^{+} H^{-}$ & $h K^{++} K^{-}$ & $H^{*} \bar{v}_{a} e_{b}$ & 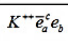 \\
\hline Hệsó́ & $\frac{-i g}{\sqrt{2}} U_{a \gamma^{*}}^{*} Y^{\mu} P_{L}$ & $\operatorname{igm}_{\pi} g^{\mu \|}$ & $\frac{i m_{v_{e}}}{2 v}$ & $\frac{i g m_{e_{g}}}{2 m_{w}}$ & $i \lambda_{1} v$ & $i \lambda_{2} v$ & $-2 i f_{c b} U_{c a}^{*} P_{L}$ & $-i g_{c b} P_{R}$ \\
\hline
\end{tabular}

Dựa vào Lagrangian toàn phần, chúng ta đưa ra các đỉnh tương tác liên quan đến quá trình rã $h \rightarrow \mu \tau$ như bảng 1 :

Bảng 1: Các đỉnh tương tác của quá trình rã $h \rightarrow \mu \tau$

Từ đó, chúng ta có thể đưa ra tất cả các giản đồ Feynman cho quá trình rã $h \rightarrow \mu \tau$ trong mô hình Zee-Babu.
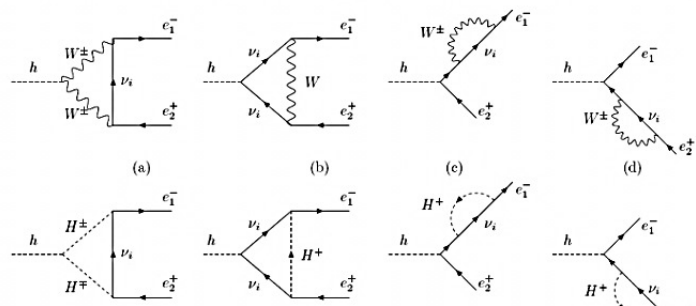
(d)

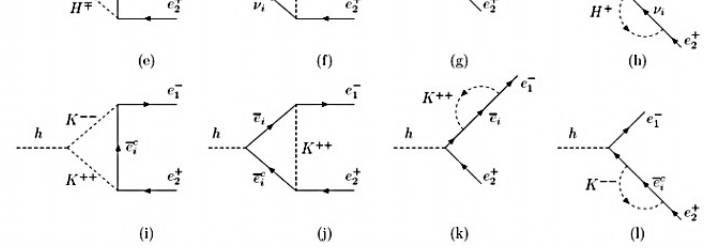

Hình 1: Các giản đồ Feynman cho quá trình rã $h \rightarrow \mu \tau$ trong mô hình Zee-Babu

Trong đó, bốn giản đồ Feynman ở hàng đầu a, b, c, $\mathrm{d}$ là đóng góp của $\mathrm{W}$ boson. Bốn giản đồ ở hàng thứ 2 là đóng góp của $H^{ \pm}$và bốn giản đồ ở hàng cuối cùng là đóng góp của $K^{ \pm \pm}$.

\subsection{Biên độ toàn phần của quá trình rã $h \rightarrow \mu T$}

Các biên độ tán xạ được tính theo các hàm $\mathrm{PV}$ (Pasarino-Veltman) lần lượt cho các giản đồ ở hình 1 . Đóng góp của $W$ boson được biểu diễn theo các hàm PV như sau: 


$$
\begin{aligned}
& M_{L}^{a}=-\frac{g^{3} m_{1}}{64 \pi^{2} m_{W}^{3}} \sum_{a=1}^{3} U_{1 a}^{*} U_{2 a}\left\{\left(2 m_{W}^{2}+m_{h}^{2}\right) m_{v_{a}}^{2} C_{0}+\right. \\
& m_{v_{a}}^{2}\left(B_{1}^{(1)}-B_{0}^{(1)}-B_{0}^{(2)}\right)-m_{2}^{2} B_{1}^{(2)} \\
& -\left[2 m_{W}^{2}\left(2 m_{W}^{2}+m_{v_{a}}^{2}+m_{1}^{2}-m_{2}^{2}\right)\right. \\
& \left.\left.+m_{v_{a}}^{2} m_{h}^{2}\right] C_{1}+\left[2 m_{W}^{2}\left(m_{1}^{2}-m_{h}^{2}\right)+m_{2}^{2} m_{h}^{2}\right] C_{2}\right\} \\
& M_{R}^{a}=-\frac{g^{3} m_{2}}{64 \pi^{2} m_{W}^{3}} \sum_{n=1}^{3} U_{1 n}^{*} U_{2 n}\left\{\left(2 m_{W}^{2}+m_{h}^{2}\right) m_{v_{n}}^{2} C_{0}\right. \\
& -m_{v_{n}}^{2}\left(B_{1}^{(2)}+B_{0}^{(1)}+B_{0}^{(2)}\right)+m_{1}^{2} B_{1}^{(1)} \\
& -\left[2 m_{W}^{2}\left(m_{2}^{2}-m_{h}^{2}\right)+m_{1}^{2} m_{h}^{2}\right] C_{1}+ \\
& \left.\left[2 m_{W}^{2}\left(2 m_{W}^{2}+m_{v_{n}}^{2}-m_{1}^{2}+m_{2}^{2}\right)+m_{v_{n}}^{2} m_{h}^{2}\right] C_{2}\right\} \\
& M_{L}^{b}=-\frac{g^{3} m_{1}}{64 \pi^{2} m_{W}^{3}} \sum_{n=1}^{3} U_{1 n}^{*} U_{2 n} m_{v_{n}}^{2}\left\{-m_{W}^{2} C_{0}\right. \\
& \left.+\left[2 m_{v_{n}}^{2}+4 m_{W}^{2}-\left(m_{1}^{2}+m_{2}^{2}\right)\right] C_{1}+B_{1}^{(1)}+B_{0}^{(12)}\right\} \\
& M_{R}^{b}=-\frac{g^{3} m_{2}}{64 \pi^{2} m_{W}^{3}} \sum_{n=1}^{3} U_{1 n}^{*} U_{2 n} m_{v_{n}}^{2}\left\{-m_{W}^{2} C_{0}\right. \\
& \left.-\left[2 m_{v_{n}}^{2}+4 m_{W}^{2}-\left(m_{1}^{2}+m_{2}^{2}\right)\right] \mathrm{C}_{2}-B_{1}^{(2)}+B_{0}^{(12)}\right\} \\
& M_{L}^{c+d}=-\frac{g^{3} m_{1}}{64 \pi^{2} m_{W}^{3}} \sum_{n=1}^{3} U_{1 n}^{*} U_{2 n} \frac{m_{2}^{2}}{m_{2}^{2}-m_{1}^{2}}\left[m_{1}^{2} B_{1}^{(1)}+m_{2}^{2} B_{1}^{(2)}+\right. \\
& \left.\left(2 m_{W}^{2}+m_{v_{n}}^{2}\right)\left(B_{1}^{(1)}+B_{1}^{(2)}\right)-2 m_{v_{n}}^{2}\left(B_{0}^{(1)}-B_{0}^{(2)}\right)\right] \\
& M_{R}^{c+d}=-\frac{g^{3} m_{2}}{64 \pi^{2} m_{W}^{3}} \sum_{n=1}^{3} U_{1 n}^{*} U_{2 n} \frac{m_{1}^{2}}{m_{3}^{2}-m_{1}^{2}}\left[m_{1}^{2} B_{1}^{(1)}+m_{2}^{2} B_{1}^{(2)} .\right. \\
& \left.+\left(2 m_{W}^{2}+m_{v_{n}}^{2}\right)\left(B_{1}^{(1)}+B_{1}^{(2)}\right)-2 m_{v_{n}}^{2}\left(B_{0}^{(1)}-B_{0}^{(2)}\right)\right)
\end{aligned}
$$

Trong đó, các hàm $C_{i}, i=0,1,2$ là hữu hạn, phần phân kỳ nằm trong các hàm $B_{0}^{(12)} ; B_{0}^{(\mathrm{i})} ; i=1,2$ và $B_{i}^{(j)} ; i=1,2 ; j=1,2$.

2.4.Khử phân kỳ trong biên độ toàn phần của quá trình $r \tilde{a} h \rightarrow \mu T$

Đóng góp của $\mathrm{W}$ boson cho quá trình rã được thể hiện tất cả trong bốn giản đồ Feynman a, b, c, d.

$$
B_{0}^{(1)}=\frac{(2 \pi \mu)^{4-D}}{i \pi^{2}} \int \frac{d^{D} k}{D_{0} D_{1}} \text { với } D=4-2 \varepsilon
$$

Thực hiện tham số hóa Feynman

$$
\begin{gathered}
\frac{1}{D_{o} D_{1}}=\int_{0}^{1} \frac{d x}{\left(k^{2}-\Delta_{1}\right)^{2}} \text { với } \\
\Delta_{1}=p_{1}^{2} x^{2}-\left(M_{0}^{2}+p_{1}^{2}-M_{1}^{2}\right) x+M_{0}^{2}-i \delta(18)
\end{gathered}
$$

Kết quả nhận được sau khi tính tích phân.

$$
\begin{aligned}
& B_{0}^{(1)}=\frac{(2 \pi \mu)^{4-D}}{i \pi^{2}} \int_{0}^{1} d x \int \frac{d^{D} k}{\left(k^{2}-\Delta_{1}\right)^{2}}=\Delta_{\varepsilon}-\int_{0}^{1} \ln \Delta_{1} d x \quad \text { với } \\
& \Delta_{\varepsilon}=\frac{1}{\varepsilon}+\ln 4 \pi \mu^{2}-\gamma_{E}
\end{aligned}
$$

Số hạng $\Delta_{\varepsilon} \rightarrow \infty$ khi $\varepsilon \rightarrow 0$, do đó phần phân kỳ của $B_{0}^{(1)}$ là

$$
\operatorname{Div}\left(B_{0}^{(1)}\right)=\Delta_{\varepsilon}
$$

Tương tự, phần phân kỳ trong các hàm $B_{0}^{(2)}, B_{1}^{(1)}$, $B_{1}^{(12)}, B_{1}^{(2)}, B_{2}^{(12)}$ được đưa ra là:

$$
\begin{aligned}
& \operatorname{Div}\left(B_{0}^{(2)}\right)=\operatorname{Div}\left(B_{0}^{(12)}\right)=\Delta_{\varepsilon} ; \\
& \operatorname{Div}\left(B_{1}^{(1)}\right)=\operatorname{Div}\left(B_{1}^{(12)}\right)=\frac{1}{2} \Delta_{\varepsilon} ; \\
& \operatorname{Div}\left(B_{1}^{(2)}\right)=\operatorname{Div}\left(B_{2}^{(12)}\right)=-\frac{1}{2} \Delta_{\varepsilon}
\end{aligned}
$$

Khi xét đến tổng đóng góp của các giản đồ có $\mathrm{W}$ boson bao gồm 4 giản đồ $\mathrm{a}, \mathrm{b}, \mathrm{c}, \mathrm{d}$, các phần trái và phải tương ứng của biên độ tán xạ là:

$$
M_{L}^{W}=\sum_{i=a, b, c, d} M_{L}^{i} ; M_{R}^{W}=\sum_{i=a, b, c, d} M_{R}^{i}
$$

Sử dụng (21), ta có thể kiểm tra số hạng có chứa phân kỳ của các phần trái và phải tương ứng của các biên độ tán xạ này là:

$$
\begin{aligned}
& \operatorname{Div}\left(M_{L}^{a}\right)=-\frac{g^{3} m_{1}}{64 \pi^{2} m_{W}^{3}} \sum_{a=1}^{3} U_{1 a}^{*} U_{2 a}\left[\Delta_{\varepsilon}\left(-\frac{3}{2} m_{v_{a}}^{2}+\frac{1}{2} m_{2}^{2}\right)\right] \\
& \operatorname{Div}\left(M_{R}^{a}\right)=-\frac{g^{3} m_{2}}{64 \pi^{2} m_{W}^{3}} \sum_{a=1}^{3} U_{1 a}^{*} U_{2 a}\left[\Delta_{\varepsilon}\left(-\frac{3}{2} m_{v_{a}}^{2}+\frac{1}{2} m_{1}^{2}\right)\right] \\
& \operatorname{Div}\left(M_{L}^{b}\right)=-\frac{g^{3} m_{1}}{64 \pi^{2} m_{W}^{3}} \sum_{a=1}^{3} U_{1 a}^{*} U_{2 a}\left(\frac{3}{2} m_{v_{a}}^{2} \Delta_{\varepsilon}\right)(23) \\
& \operatorname{Div}\left(M_{R}^{b}\right)=-\frac{g^{3} m_{2}}{64 \pi^{2} m_{W}^{3}} \sum_{a=1}^{3} U_{1 a}^{*} U_{2 a}\left(\frac{3}{2} m_{v_{a}}^{2} \Delta_{\varepsilon}\right) \\
& \operatorname{Div}\left(M_{L}^{c+d}\right)= \\
& -\frac{g^{3} m_{1}}{64 \pi^{2} m_{W}^{3}} \sum_{a=1}^{3} U_{1 a}^{*} U_{2 a}\left\{\frac{m_{2}^{2}}{m_{2}^{2}-m_{1}^{2}}\left[\frac{1}{2} \Delta_{\varepsilon}\left(m_{1}^{2}-m_{2}^{2}\right)\right]\right\} \\
& \operatorname{Div}\left(M_{L}^{c+d}\right)= \\
& -\frac{g^{3} m_{2}}{64 \pi^{2} m_{W}^{3}} \sum_{a=1}^{3} U_{1 a}^{*} U_{2 a}\left\{\frac{m_{1}^{2}}{m_{2}^{2}-m_{1}^{2}}\left[\frac{1}{2} \Delta_{\varepsilon}\left(m_{1}^{2}-m_{2}^{2}\right)\right]\right\}
\end{aligned}
$$

Phần trái và phần phải của biên độ này, hoàn toàn triệt tiêu phân kỳ.

$$
\begin{aligned}
& \operatorname{Div}\left(M_{L}^{W}\right)=\sum_{i=a, b, c, d} \operatorname{Div}\left(M_{L}^{i}\right)= \\
& -\frac{g^{3} m_{1}}{64 \pi^{2} m_{W}^{3}} \sum_{a=1}^{3} U_{1 a}^{*} U_{2 a}\left[\Delta_{\varepsilon}\left(-\frac{3}{2} m_{v_{a}}^{2}+\frac{1}{2} m_{2}^{2}+\frac{3}{2} m_{v_{a}}^{2}-\frac{1}{2} m_{2}^{2}\right)\right]=0
\end{aligned}
$$


$\operatorname{Div}\left(M_{R}^{W}\right)=\sum_{i=a, b, c, d} \operatorname{Div}\left(M_{R}^{i}\right)=$

$-\frac{g^{3} m_{2}}{64 \pi^{2} m_{W}^{3}} \sum_{a=1}^{3} U_{1 a}^{*} U_{2 a}\left[\Delta_{\varepsilon}\left(-\frac{3}{2} m_{v_{a}}^{2}+\frac{1}{2} m_{1}^{2}+\frac{3}{2} m_{v_{a}}^{2}-\frac{1}{2} m_{1}^{2}\right)\right]=0$

Hoàn toàn tương tự, khi xét đến đóng góp của $H^{+}$ ở bốn giản đồ Feynman e, $\mathrm{f}, \mathrm{g}, \mathrm{h}$ và đóng góp của $K^{++}$ở bốn giản đồ Feynman i, j, k, l, chúng ta cũng có thể chỉ ra các thành phần trái và các thành phần phải của các biên độ này đều được khử phân kỳ. Do đó, biên độ toàn phần là hữu hạn.

\section{Kết luận}

Trong phạm vi mô hình Zee-Babu, bài báo đạt được các kết quả sau:

Xác định các đỉnh tương tác, từ đó đưa ra tất cả các giản đồ Feynman cho quá trình rã $h \rightarrow \mu \tau$.

Tính biên độ của các giản đồ Feynman và biểu diễn theo các hàm $\mathrm{PV}$. Sử dụng phương pháp tham số hóa Feynman để tách các hàm $\mathrm{PV}$ thành các phần phân kỳ và phần hữu hạn.

Chỉ ra rằng tổng tất cả các giản đồ có đóng góp của W boson, của $H^{+}$và $K^{++}$đều lần lượt bị khử phân kỳ. Do đó biên độ toàn phần là hữu hạn.

Biên độ toàn phần của quá trình rã là hữu hạn. Đây là cơ sở để thực hiện tính số và so sánh với các dữ liệu thực nghiệm, từ đó đưa ra giới hạn các vùng không gian tham số của mô hình và đề xuất các hiện tượng Vật lý mới.

Nghiên cứu này được tài trợ từ nguồn kinh phí Khoa học \& Công nghệ của Trường ĐHSP Hà Nội 2 cho đề tài, mã số: C.2018.08

\section{TÀI LIỆ THAM KHẢO}

1. ATLAS and CMS Collaborations (2016), Measurements of the Higgs boson production and decay rates and constraints on its couplings from a combined ATLAS and CMS analysis of the LHC pp collision data at $\sqrt{s}_{s}=7$ and $8 \mathrm{TeV}$, JHEP 1608, 045;

2. C. Patrignani et al (2016). [Particle Data Group], Review of particle physics, Chinese Physics C 40, 100001;

3. Daniel Schmidt, Thomas Schwetz, He Zhang (2014), Status of the Zee-Babu model for neutrino mass and possible tests at a like-sign linear collider, Nucl. Phys. B 885, 524-541;

4. Juan Herrero-Garcia, Miguel Nebot, Nuria Rius, Arcadi Santamaria (2014), The Zee-Babu Model revisited in the light of new data, Nucl. Phys. B 885, 542-570;

5. K. H. Kiem, H.T.Hung and L.T.Hue (2016), Prog.Theor. Exp.Phys. 2016, 113B03;

6. L.T. Hue, H. N. Long, T.T.Thuc and T. Phong Nguyen (2016), Nucl.Phys. B 907, 37; Phys.Rev. D93, 115026;

7. Miguel Nebot et al, Prospects for the Zee-Babu Model at the LHC and low energy experiments, Phys.Rev.D77, 093013,2008;

8. Takaaki Nomura, Hiroshi Okada (2016), An Extended Colored Zee-Babu Model,Phys. Rev. D 94, 075021;

9. Tommy Ohlsson, Thomas Schwetz, He Zhang (2009), Non-standard neutrino interactions in the ZeeBabu model,Phys.Lett.B681:269-275.

\section{Divergent cancelling in Total scattering amplitude of $h \rightarrow \mu$ in decays Zee-Babu model}

\section{Tran Trung Hieu, Nguyen Thi Thu Van, Duong Thi Kieu Tu, Ha Thanh Hung, Trịnh Phi Hiep}

\section{Article info}

Recieved:

26/03/2018

Accepted:

$12 / 6 / 2018$

Keywords:

$P V$ functions, divergent cancelling, Zee-Babu model, lepton flavor violating, Higgs boson decays, etc.

\section{Abstract}

The Zee-Babu model continuedof Zee model, is extended from the standard model to solve mass and mixing of neutrino. Scalars of Zee-Babu model is simpler than Zee model. So the neutrino problem is solved naturally. Higgs decay channels have been tested from accelerators. New interactions of Zee-Babu model increase signal of the lepton flavor violating Higgs decay. Studying these channels help us to constrain parameter space of this model and to give new physics. 